\title{
Giant Calcified Renal Artery Aneurysm: Traditional RX versus Three-Dimensional Computed Tomography
}

\author{
Mauro Gacci ${ }^{1}$, Omar Saleh ${ }^{1}$, Annalisa Mantella ${ }^{1}$, Leonidas Azas ${ }^{2}$, Paola Romagnani ${ }^{3}$, \\ Andrea Minervini ${ }^{1}$, Sergio Serni ${ }^{1}$, Marco Carini ${ }^{1}$ \\ ${ }^{1}$ Department of Urology, University of Florence, Florence, Italy \\ ${ }^{2}$ Department of Vascular Surgery, University of Florence, Florence, Italy \\ ${ }^{3}$ Excellence Center for Research, Transfer and High Education (DENOTHE), University of Florence, Florence, Italy \\ Email: os11nov@hotmail.com
}

Received November 7, 2012; revised December 16, 2012; accepted December 26, 2012

\begin{abstract}
A 65-year-old woman with no history of previous flank trauma, renal stone or upper urinary tract infections, presented for flank pain and left hydro-uretero-nephrosis seven days after hysterectomy. Percutaneous pielography revealed narrowing of the distal ureter, without endoureteral mass. The plain abdomen film incidentally showed a 3-cm calcified ring on the left renal shadow, who resulted external to the collecting system at pielography. A 3-dimensional-CT scan with angiographic reconstruction revealed a 3-cm calcified renal artery aneurysm. The vascular surgeon suggested a watchful waiting. The patient underwent ureteral reimplantation with ureteral stenting, allowing a complete recovery of iatrogenic stenosis two months postoperatively.
\end{abstract}

Keywords: Renal Artery Aneurysm; 3D-CT; Angiographic Reconstruction

\section{Introduction}

Renal artery aneurysms have been encountered with increasing frequency over the past decade. It is slightly more common in women than men and in the right than left renal artery [1]. Many cases are asymptomatic and found incidentally, and their occurrence has been recently increasing with the advancement of imaging techniques. Angiography is the gold standard in the diagnosis of renovascular injuries, and it has the additional advantage to possesses the potential of therapeutic intervention [2]. Therefore, after any invasive urological procedure, CT angiography can be considered as the first choice for renal artery injury $[3,4]$. In the present case, we incidentally discovered a lesion of the left renal artery and we completed the diagnostic work-up with a 3D-CT scan.

\section{Case Report}

A 67-year-old woman was referred to the Department of Urology for left flank pain developed seven days after hysterectomy for uterine leiomyomatosis. The patient had no history of previous flank trauma, renal stone or upper urinary tract infections. Physical examination was unremarkable, with only minimal flank pain at Giordano manoeuvre; body temperature was $37^{\circ} \mathrm{C}$, blood pressure was $120 / 75 \mathrm{mmHg}$. White cell count was within the limits $\left(9 \times 10^{9}\right.$ white blood cells in a litre of blood); renal and liver functions were normal (creatinine $0.9 \mathrm{mg} / \mathrm{dL}$, total bilirubin $0.8 \mathrm{mg} / \mathrm{dL}$ ). A severe left hydronephrosis was detected at ultrasound, with no sign of uretheral stones or masses. The plain abdomen film showed a 3-cm calcified ring on the left renal shadow (Figure 1(a)). Percutaneous pielography confirmed hydronephrosis, and showed no relationship of the calcified mass with the collecting system (Figure 1(b)).

A 3-dimensional-CT scan revealed a 3-cm round hollow mass with calcified walls located in front of anterior renal surface. The lesion had no connection with renal pelvis (Figure 2(a)) but was firmly related with renal artery, and it was compatible with calcified renal artery aneurysm (Figure 2(b)). The patient was treated with an open access ureteral reimplantation performed on the suture of the previous surgical approach. A direct uretheral reimplantation on the upper bladder wall was performed, with psoas hitch and double J uretheral stenting. At the 1 month follow up visit there was a complete recovery of the obstruction. The vascular surgeon suggested a watchful waiting by monitoring blood pressure, renal function, and imaging every 6 months.

\section{Discussion}

Renal artery aneurysms (RAA) are rare, with an estimated incidence below 1\%. Hypertension and fibro-muscular disease of the renal artery are the leading classes of 


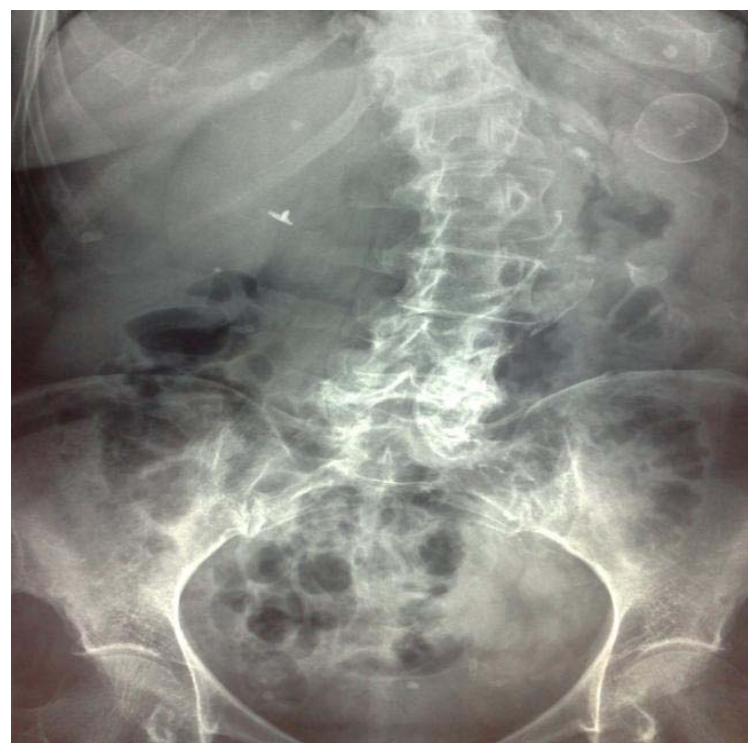

(a)

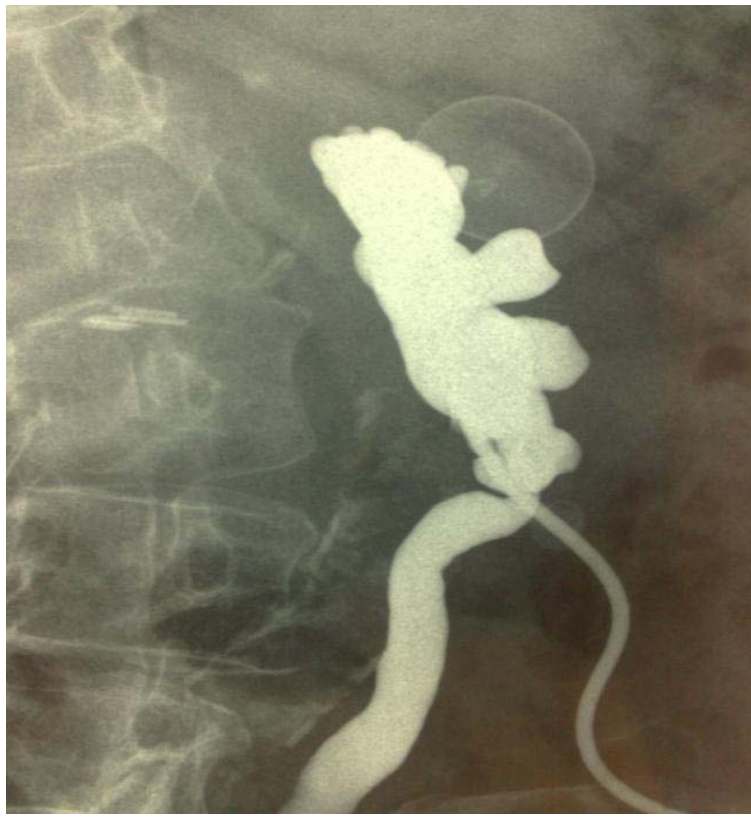

(b)

Figure 1. (a) Plain abdomen film performed showing a left 3-cm calcified ring on the left renal shadow; (b) Pielography demonstrating no relationships of the calcified mass with the collecting system.

risk [5]. There is no significant difference in side presentation (right side in $43 \%$, left in $36 \%$ and $21 \%$ bilateral). Aneurysm usually involve the main renal artery or the primary branches. Mean size at diagnosis ranges from 0.5 to $8 \mathrm{~cm}$, with a mean diameter of $2.1 \mathrm{~cm}$. Degenerative (arteriosclerosis, fibrodysplasia), inflammatory (arteritis) or traumatic diseases, including iatrogenic damages after kidney puncture [5], may cause RAA.

RAA can be classified into 4 categories: 1) true macroaneurysm; 2) aneurysmal dissections; 3) fusiform micro-

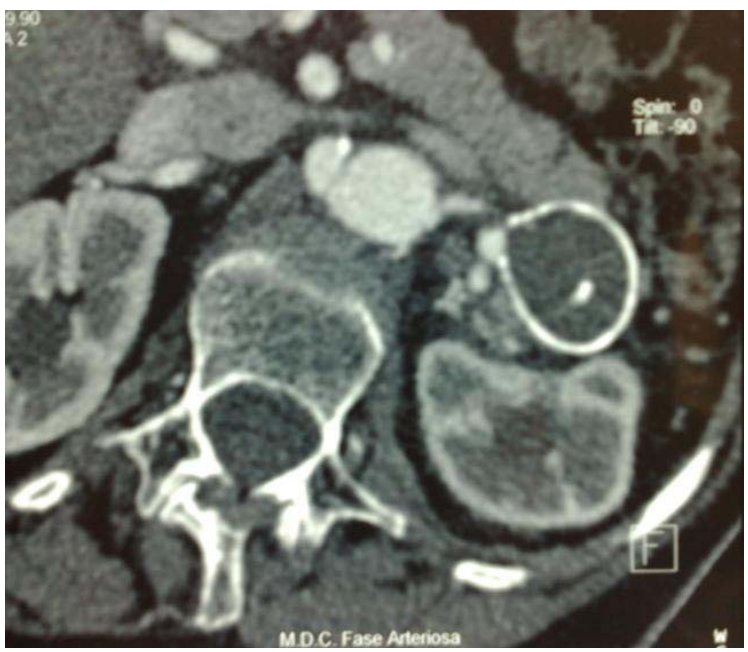

(a)

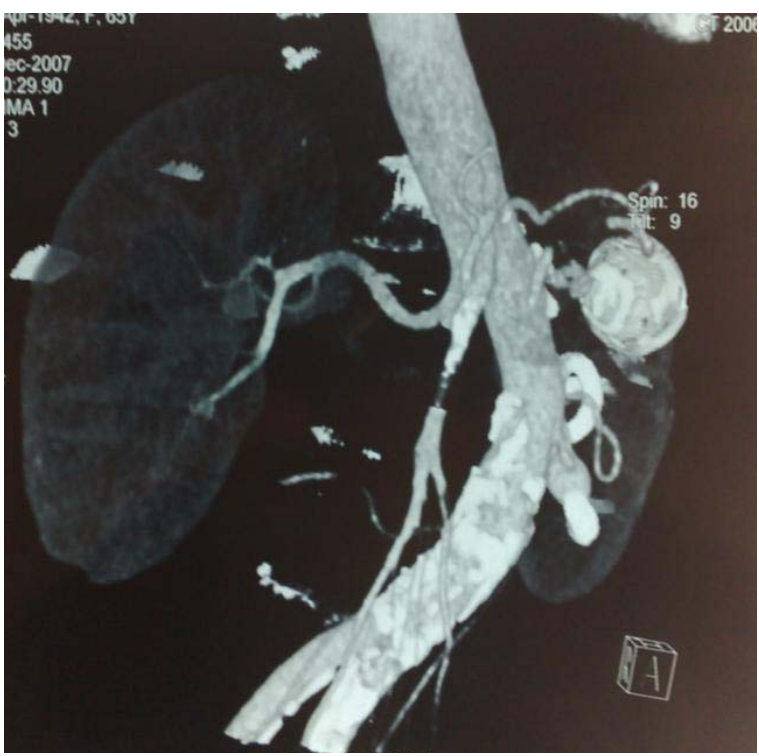

(b)

Figure 2. (a) CT scan showing a calcified round mass facing anterior renal surface; (b) 3-dimensional TC re-construction showing the relationship with renal artery, compatible with calcified renal artery aneurysm.

aneurysmal dilations; and 4) microaneurysm resulting from arteritis [6]. They are usually asymptomatic and serum creatinine levels are mostly within normal range at diagnosis. Complications, including renovascular hypertension, renal embolization with infarction, thrombosis or dissection, and arteriovenous fistula, [5] are rarely reported.

\section{Conclusion}

The diagnosis has been recently increased by the spreading use of imaging techniques [7]. Conventional renal angiography represents the gold standard in the detection of renal aneurysms, though the spiral CT renal angio- 
graphy with three dimensional reconstruction (3D-CT) adds the benefits of being quicker, more costeffective, and non-invasive. Moreover, the use of 3D-CT workstation allows accurate treatment planning, especially for endovascular procedures [8].

Management options include observation, trans-catheter-occlusion or surgical intervention. The indications for surgery include: symptomatic or enlarging aneurysms, renal embolization, aneurysms in pregnant females or those considering pregnancy, renovascular hypertension, aneurysms $>2.5 \mathrm{~cm}[9]$.

\section{REFERENCES}

[1] J. C. Stanley, L. M. Messina and G. B. Zelenock, "Splanckmc and Renal Artery Aneurysms," In: W. S. Moore, Ed., Vascular Surgery: A Comprehensive Review, WB Saunders, Philadelphia, 1491, pp. 335-349.

[2] Y. H. Rokni and Y. Moharamzad, "Endovascular Treatment of Renal Arteriovenous Fistula Following a Stab Wound," Urology Journal, Vol. 5, No. 2, 2008, pp. 129131.

[3] L. J. Zhang, G. F. Yang, J. Qi and W. Shen, "Renal Artery Aneurysm: Diagnosis and Surveillance with MultiDetector Row Computed Tomography,” Acta Radiologica, Vol. 48, No. 3, 2007, pp. 274-279. doi:10.1080/02841850601161521
[4] F. Y. Dönmez, M. Cokun, A. Uyuur, et al., "Non-Invasive Imaging Findings of Idiopathic Renal Arteriovenous Fistula,” Diagnostic and Interventional Radiology, Vol. 14, No. 2, 2008, pp. 103-105.

[5] A. Lumsden, T. Salam and K. Walton, "Renal Artery Aneurysm: A Report of 28 Cases,” Cardiovascular Surgery, Vol. 4, No. 2, 1996, pp. 185-189. doi:10.1016/0967-2109(96)82312-X

[6] J. C. Stanley, E. L. Rhodes, B. L. Cewertz, C. Y. Chang, J. F. Walter and W. J. Fry, "Renal Artery Aneurysms: Significance of Macroaneurysms Exclusive of Dissections and Fibrodysplastic Mural Dilations," Archives of Surgery, 1975; Vol. 110, No. 11, 1975, pp. 1327-1333. doi:10.1001/archsurg.1975.01360170067009

[7] S. P. Stanl and E. Fishman, "Three Dimensional Ct Angiography: Renal Applications,” Semin Ultrasound CT MR, Vol. 19, No. 5, 1998, pp. 413-424. doi:10.1016/S0887-2171(98)90018-X

[8] W. Higashiura, S. Sakaguchi, N. Tabayashi, S. Taniguchi and K. Kichikawa, "Impact of 3-Dimensional-Computed Tomography Workstation for Precise Planning of Endovascular Aneurysm Repair,” Circulation Journal, Vol. 72, No. 12, 2008, pp. 2028-2034. doi:10.1253/circj.CJ-08-0331

[9] C. Dzsinich, P. Gloviczki, M. A. McKusick, P. C. Pairolero, T. C. Bower, J. W. Hallett Jr. and K. J. Cherry Jr., "Surgical Management of Renal Artery Aneurysm," Cardiovascular Surgery, Vol. 1, No. 3, 1993, pp. 243-247.

\section{Abbreviation and Acronym}

Renal artery aneurysms (RAA) Units 